\title{
ON ANGLES IN CERTAIN METRIC SPACES*
}

\author{
BY W. A. WILSON
}

1. Introduction. In a series of articles on metrical geometry $\dagger$ Menger has made a study of the geometry of certain abstract metric spaces and in particular he has obtained conditions for the congruence of metric spaces with sub-sets of euclidean spaces. In a recent article $\ddagger$ he suggests a system of axioms for "angle spaces" and related problems.

It will be shown in this note that a theory of angles analogous to that of euclidean space is possible for convex complete metric spaces any four points of which are congruent with four points in some euclidean space. From this certain theorems regarding tangents to simple arcs are deduced.

2. Notation and Definitions. A euclidean space of $n$ dimensions will be denoted by $E_{n}$.

If $a$ and $b$ are two points, the symbol $a b$ will denote the distance between them or, at times, the straight line segment joining them.

If, corresponding to a set $A$, there is a set $A^{\prime}$, in some $E_{n}$ congruent to $A$, we say that $A$ can be imbedded in $E_{n}$. The word congruence has its usual meaning: $A$ is congruent to $A^{\prime}$ if there is a one to one correspondence $x \sim x^{\prime}$ between the points of $A$ and $A^{\prime}$ such that, if $x \sim x^{\prime}$ and $y \sim y^{\prime}$, then $x y=x^{\prime} y^{\prime}$. The congruence of $A$ to $A^{\prime}$ is denoted by $A \cong A^{\prime}$.

In stating a congruence between two finite sets it will be understood that the pairs of points correspond in the order written. Thus, in the congruence $a+b+c \cong a^{\prime}+b^{\prime}+c^{\prime}$, we have $a \sim a^{\prime}$, $b \sim b^{\prime}$, and $c \sim c^{\prime}$; the congruence $a+b+c \cong a^{\prime}+c^{\prime}+b^{\prime}$ is a different congruence.

Likewise, if $a b$ and $a^{\prime} b^{\prime}$ denote two simple arcs, $a b \cong a^{\prime} b^{\prime}$ if there is a one to one correspondence between their points such

* Presented to the Society, March 25, 1932.

$\dagger$ Untersuchungen über allgemeine Metrik, Mathematische Annalen, vol. 100, pp. 75-163, and vol. 103, pp. 466-501.

$\ddagger$ Some applications of point-set methods, Annals of Mathematics, vol. 32, pp. 739-760. 
that $a \sim a^{\prime}, b \sim b^{\prime}$, and the relations $x \sim x^{\prime}$ and $y \sim y^{\prime}$ imply that $x y=x^{\prime} y^{\prime}$. As in the previous paragraph the congruences $a b \cong a^{\prime} b^{\prime}$ and $a b \cong b^{\prime} a^{\prime}$ are different.

If any four points of a space $Z$ can be imbedded in a euclidean space, we shall say for brevity that $Z$ has the four-point property.* Obviously, in that case any four points can be imbedded in $E_{3}$.

The terms convex and externally convex are used in the sense of Menger. (See Annals of Mathematics, loc. cit., p. 742.)

3. Two Lemmas. As a preliminary we prove two lemmas which form the basis of most of the theorems following.

Lemma 1. Let $a, b, c$, and $d$ be four points of a metric space which are congruent to four points of some euclidean space. If $a^{\prime}$, $b^{\prime}$, and $c^{\prime}$ are points in some $E_{3}$ such that $a+b+c \cong a^{\prime}+b^{\prime}+c^{\prime}$, there is a point $d^{\prime}$ in this $E_{3}$ such that $a+b+c+d \cong a^{\prime}+b^{\prime}+c^{\prime}+d^{\prime}$.

PROOF. By hypothesis $a+b+c+d \cong a^{\prime \prime}+b^{\prime \prime}+c^{\prime \prime}+d^{\prime \prime}$, where $a^{\prime \prime}, b^{\prime \prime}, c^{\prime \prime}$, and $d^{\prime \prime}$ lie in some $E_{3}$. If they do not lie in an $E_{2}$, they are the vertices of a tetrahedron $T^{\prime \prime}$. One face of this is the triangle $a^{\prime \prime} b^{\prime \prime} c^{\prime \prime}$, which is congruent to thet riangle $a^{\prime} b^{\prime} c^{\prime}$. Hence, by euclidean geometry, $T^{\prime \prime}$ is congruent to a tetrahedron $T^{\prime}=a^{\prime} b^{\prime} c^{\prime} d^{\prime}$ in the $E_{3}$ under discussion. This gives at once $a+b+c+d \cong a^{\prime}+b^{\prime}+c^{\prime}+d^{\prime}$. The special cases where $a^{\prime \prime}, b^{\prime \prime}, c^{\prime \prime}$, and $d^{\prime \prime}$ lie in an $E_{1}$ or in an $E_{2}$ are treated in like manner.

Lemma 2. Let $Z$ be a metric space which has the four-point property. Let $a$ and $b$ be any two points and $k$ be any positive constant. Then there is at most one point $c$ for which $a c=k \cdot a b$ and (a) $a c+c b=a b$; (b) $a b+b c=a c$; or (c) $c a+a b=c b$.

Proof. In (a) $k \leqq 1$; in (b) $k \geqq 1$; and in (c) $k$ may have any value. We prove (b), which is typical. Suppose that for some $k$ there were two points $c$ and $d$ satisfying the hypotheses. Now the four points $a, b, c$, and $d$ are congruent to points $a^{\prime}, b^{\prime}, c^{\prime}$, and $d^{\prime}$ in some $E_{3}$. Since $a^{\prime} b^{\prime}+b^{\prime} c^{\prime}=a^{\prime} c^{\prime}$, the points $a^{\prime}, b^{\prime}$, and $c^{\prime}$ lie on a line $L$. Since $a^{\prime} b^{\prime}+b^{\prime} d^{\prime}=a^{\prime} d^{\prime}$, the points $a^{\prime}, b^{\prime}$, and $d^{\prime}$ lie on a line $L^{\prime}$. As $L \cdot L^{\prime} \supset a^{\prime}+b^{\prime}$, the lines $L$ and $L^{\prime}$ are identical.

* A simple example of a metric space not having the four-point property is the surface of a euclidean sphere, with the distance between any two points defined as the length of the shorter great circle arc joining them. Another example is given in $\$ 12$, where the set $e+e_{1}+e_{2}+f$ cannot be imbedded in euclidean space. 
Since $a^{\prime} c^{\prime}$ and $a^{\prime} d^{\prime}$ are greater than $a^{\prime} b^{\prime}$ and $b^{\prime} c^{\prime}$ or $b^{\prime} d^{\prime}$, respectively, by (b), it follows that $c^{\prime}$ and $d^{\prime}$ lie on $a^{\prime} b^{\prime}$ produced beyond $b^{\prime}$. Since $a^{\prime} c^{\prime}=k \cdot a^{\prime} b^{\prime}=a^{\prime} d^{\prime}$, we have $c^{\prime}=d^{\prime}$. Hence $c=d$.

4. Collinear Points. If $a$ and $b$ are two points and $c$ is a third point satisfying one of the three conditions of Lemma 2 above, we say that $c$ is collinear with $a$ and $b$. In other words three points are collinear if one lies between the other two.

If the space is complete and convex, there is for each $k$ between 0 and 1 at least one point $c$ for which $a b=a c+c b$ and $a c=k \cdot a b$. (See Mathematische Annalen, loc. cit., p. 89.) Likewise, if it is also externally convex, there is at least one point $c$ for which (b) and (c) in Lemma 2 are valid.

Consequently, if we define a segment, ray, or open line as a set of points congruent with a euclidean segment, ray, or open line, respectively, we have the following results from Menger's work and Lemma 2 :

Theorem 1. If a convex complete space $Z$ has the four-point property, there is one and only one segment joining any pair of points $a$ and $b$. This is the set $\{x\}$ for which $a b=a x+x b$.

THEOREM 2. If a convex complete space $Z$ has the four-point property, the set of all points collinear with any two points is a segment, ray, or open line.

If the two points in Theorem 2 are $a$ and $b$, we call the set of collinear points the line determined by $a$ and $b$. The line will be an open line if $Z$ is both convex and externally convex; otherwise it may be bounded at one or both ends. It is an easy deduction that a line is determined by any two of its points, and that two lines can have but one point in common.

5. ThEOREM 3. Let $Z$ be a convex complete space which has the four-point property. Let $L, M$, and $N$ be three different lines in $Z$ having one point a in common. Then $L+M+N$ can be imbedded in $E_{3}$.

Proof. Let $b, c$, and $d$ lie on $L, M$, and $N$, respectively. By hypothesis, $a, b, c$, and $d$ are congruent to points $a^{\prime}, b^{\prime}, c^{\prime}$, and $d^{\prime}$ in some $E_{3}$. This congruence defines congruences of $L, M$, and $N$, respectively, to $L^{\prime}, M^{\prime}$, and $N^{\prime}$, which are euclidean segments, rays, or open lines through $a^{\prime}$ and $b^{\prime}, a^{\prime}$ and $c^{\prime}$, and $a^{\prime}$ and $d^{\prime}$, re- 
spectively. Obviously $L^{\prime} \cdot M^{\prime}=L^{\prime} \cdot N^{\prime}=M^{\prime} \cdot N^{\prime}=a^{\prime}$. For any point $x$, say on $L$, there is a unique corresponding point $x^{\prime}$ on $L^{\prime}$ given by the congruence $L \cong L^{\prime}$. To show that $L+M+N$ $\cong L^{\prime}+M^{\prime}+N^{\prime}$, we must prove that, if $x \sim x^{\prime}$ and $y \sim y^{\prime}$, then $x y=x^{\prime} y^{\prime}$.

To fix the ideas let $x$ lie on $L$ and $y$ on $M$. By the hypothesis and Lemma 1 , there is a point $x^{\prime \prime}$ such that $a+b+c+x \cong a^{\prime}+b^{\prime}$ $+c^{\prime}+x^{\prime \prime}$. Of the points $a, b$, and $x$, one lies between the other two, say $b$ between $a$ and $x$. Then $a x=a b+b x$ and $a^{\prime} x^{\prime \prime}=a^{\prime} b^{\prime}$ $+b^{\prime} x^{\prime \prime}$. Thus $a^{\prime} x^{\prime \prime}=a x$ and $x^{\prime \prime}$ lies on $a^{\prime} b^{\prime}$ produced. By the congruence $L \cong L^{\prime}, a^{\prime} x^{\prime}=a^{\prime} b^{\prime}+b^{\prime} x^{\prime}$ and $x^{\prime}$ lies on $a^{\prime} b^{\prime}$ produced. Since $a^{\prime} x^{\prime}=a x=a^{\prime} x^{\prime \prime}$, we have $x^{\prime}=x^{\prime \prime}$. Thus $a+b+c+x \cong a^{\prime}$ $+b^{\prime}+c^{\prime}+x^{\prime}$.

Again applying the four-point hypothesis and Lemma 1, we have a point $y^{\prime \prime}$ such that $a+c+x+y \cong a^{\prime}+c^{\prime}+x^{\prime}+y^{\prime \prime}$. To fix the ideas, let $y$ lie between $a$ and $c$. Then $a y+y c=a c$ and $a^{\prime} y^{\prime \prime}+y^{\prime \prime} c^{\prime}=a^{\prime} c^{\prime}$. By the congruence $M \cong M^{\prime}, a^{\prime} y^{\prime}+y^{\prime} c^{\prime}=a^{\prime} c^{\prime}$. Since $a^{\prime} y^{\prime}=a^{\prime} y^{\prime \prime}$ and $y^{\prime \prime} c^{\prime}=y^{\prime} c^{\prime}$, we have $y^{\prime}=y^{\prime \prime}$. But $x y=x^{\prime} y^{\prime \prime}$; this gives $x y=x^{\prime} y^{\prime}$, which was to be proved.

COROLlARY. Under the above hypotheses two intersecting lin:; can be imbedded in a plane.

6. TheOREM 4. Let $Z$ be a convex complete space which has the four-point property. Let $a, b$, and $c$ be non-collinear points in $Z$ which are congruent to the points $a^{\prime}, b^{\prime}$, and $c^{\prime}$ in some $E_{2}$. Then* $a b+b c+c a \cong a^{\prime} b^{\prime}+b^{\prime} c^{\prime}+c^{\prime} a^{\prime}$.

Proof. The given congruence defines congruences $a b \cong a^{\prime} b^{\prime}$, $b c \cong b^{\prime} c^{\prime}$, and $c a \cong c^{\prime} a^{\prime}$; and therefore a one to one correspondence between the points $\{x\}$ of $a b+b c+c a$ and the points $\left\{x^{\prime}\right\}$ of $a^{\prime} b^{\prime}+b^{\prime} c^{\prime}+c^{\prime} a^{\prime}$. We must prove that, if $x \sim x^{\prime}$ and $y \sim y^{\prime}$, then $x y=x^{\prime} y^{\prime}$.

To fix the ideas let $x$ lie on $a c$ and $y$ on $b c$. By the four-point hypothesis and Lemma 1 , there is a point $y^{\prime \prime}$ in the space containing $E_{2}$ such that $a+b+c+y \cong a^{\prime}+b^{\prime}+c^{\prime}+y^{\prime \prime}$. Now $b^{\prime} y^{\prime \prime}$ $+y^{\prime \prime} c^{\prime}=b^{\prime} c^{\prime}$ and $b^{\prime} y^{\prime}+y^{\prime} c^{\prime}=b^{\prime} c^{\prime}$. Since $b^{\prime} y^{\prime \prime}=b y=b^{\prime} y^{\prime}$ and $y^{\prime \prime} c^{\prime}$ $=y c=y^{\prime} c^{\prime}$, we have $y^{\prime \prime}=y^{\prime}$.

* This congruence is a congruence between the two sets of three segments; it does not imply anything regarding the set of points lying on segments joining $a$ to points of the segment $b c$ and the point set forming the interior of the euclidean triangle $a^{\prime} b^{\prime} c^{\prime}$. 
Repeating this reasoning, we find that there is an $x^{\prime \prime}$ such that $a+c+y+x \cong a^{\prime}+c^{\prime}+y^{\prime}+x^{\prime \prime}$. Then $a^{\prime} x^{\prime \prime}+x^{\prime \prime} c^{\prime}=a x+x c$ $=a c=a^{\prime} c^{\prime}$ and $a^{\prime} x^{\prime}+x^{\prime} c^{\prime}=a x+x c=a c=a^{\prime} c^{\prime}$. Hence $x^{\prime}=x^{\prime \prime}$. Therefore $x y=x^{\prime \prime} y^{\prime}=x^{\prime} y^{\prime}$, which was to be proved.

7. Angle between two Lines. In consequence of the two preceding sections the angle between two lines in a convex complete space having the four-point property can be identified with the euclidean angle between their congruent images. Thus, if $a, b$, and $c$ are the vertices of a "triangle," the cosine of the angle $b a c$ is

$$
\frac{(a b)^{2}+(a c)^{2}-(b c)^{2}}{2(a b)(a c)} .
$$

With regard to such questions as tangency to a simple arc we are handicapped by the absence of a co: rdinate system and the fact that it may be impossible to imbed any more than four points of the arc in any one euclidean space. There is, however, no difficulty in defining tangents.

Let $C$ be a simple arc with its points ordered from left to right and let $a$ be one of its points. Let $L$ be a half-line having $a$ as one end and passing through a point $x$ at the right (left) of $a$ on $C$, and let $T$ be a half-line from $a$ passing through some point $t$. If the angle $x a t$ approaches 0 as $x \rightarrow a$, we say that $T$ is a righthand (left-hand) tangent to $C$ at $a$. In consequence of $\$ 5$ and the properties of euclidean space angles obey the rule that angle $b a c+$ angle $b a d \geqq$ angle $c a d$; hence there cannot be more than one right-hand or left-hand tangent at a point.

If $S$ is a left-hand and $T$ a right-hand tangent at $a$, there are three possibilities: a cusp, an angle-point, or an ordinary tangent. The first occurs when the angle between $S$ and $T$ is zero, or $S=T$; the last occurs when $S$ and $T$ make an angle of $\pi$, or $S+T$ is a line; and we have an angle-point when the angle between $S$ and $T$ is neither 0 nor $\pi$.

To obtain necessary and sufficient conditions for the existence of tangents we need the following lemma from plane trigonometry.

8. Lemma 3. Let axy be a triangle congruent with a plane triangle and $a x \leqq a y$. In order that angle $x a y \rightarrow 0$ as $a x+a y \rightarrow 0$ it is necessary and sufficient that $(a x+x y-a y) /(a x) \rightarrow 0$; in order that 
angle $x a y \rightarrow \pi$ as ax+ay $\rightarrow 0$ it is necessary and sufficient that $(a x+a y-x y) /(a x) \rightarrow 0$.

Proof. Let $\theta$ be the angle $x a y$. Then by the law of cosines

$$
1-\cos \theta=\frac{(a x+x y-a y)(a y+x y-a x)}{2(a x)(a y)} .
$$

Now $\theta \rightarrow 0$ if and only if $1-\cos \theta \rightarrow 0$. Since $|x y-a x| \leqq a y$, it is clear that $1-\cos \theta \leqq(a x+x y-a y) /(a x)$; hence the condition is sufficient.

To prove the converse we set $(a x+x y-a y) /(a x)=m$. Then $m$ is a non-negative function of the sides of the triangle, and

$$
a x+x y-a y=m \cdot a x .
$$

Now $2(a y-a x) \geqq 0$. Adding this to (2), we have $a y+x y-a x$ $\geqq m \cdot a x$, and (1) gives

$$
1-\cos \theta \geqq \frac{m^{2} \cdot a x}{2 \cdot a y} .
$$

Since $1-\cos \theta \rightarrow 0$, either $m \rightarrow 0$ or there is a sequence of triangles $\left\{a x_{i} y_{i}\right\}$ for which $a x_{i} \leqq a y_{i}, a x_{i} \rightarrow 0$, and $a y_{i} \rightarrow 0$ as $i \rightarrow \infty$, and $m$ has a positive lower bound $k$. Then $a x_{i} / a y_{i} \rightarrow 0$ and for $i$ large enough the relation $a x_{i}+x_{i} y_{i} \geqq a y_{i}$ gives $x_{i} y_{i} \geqq a x_{i}$, or

$$
\frac{a y_{i}+x_{i} y_{i}-a x_{i}}{2 \cdot a y_{i}} \geqq \frac{1}{2} .
$$

But then (1) and (2) give

$$
1-\cos \theta \geqq k / 2 \text {, }
$$

which is a contradiction. Hence the first part of the theorem is proved.

For the second part we must examine $1+\cos \theta$, which approaches 0 as $\theta \rightarrow \pi$. Now

$$
1+\cos \theta=\frac{(a x+a y+x y)(a x+a y-x y)}{2(a y)(a x)} .
$$

Since $x y \leqq a x+a y, \quad a x+a y+x y \leqq 2(a x+a y) \leqq 4(a y)$. Thus we have $1+\cos \theta \rightarrow 0$ if $(a x+a y-x y) /(a x) \rightarrow 0$. On the other hand, we have $(a x+a y+x y) /[2(a y)]>1 / 2$; hence the condition is necessary. 
9. TheOREM 5. Let $C$ be a simple arc in a convex complete space $Z$ having the four-point property. Let $C$ have a right-hand (lefthand) tangent at a point $a$ and let $x$ and $y$ be points of $C$ at the

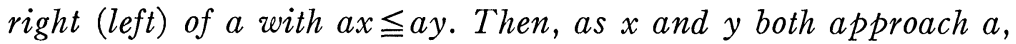
$(a x+x y-a y) /(a x) \rightarrow 0$.

Proof. Let $T$ be the tangent and $t$ be a point of $T$ different from $a$. By the definition of a unilateral tangent, angle $\operatorname{tax} \rightarrow 0$ as $x \cdot \rightarrow a$ and angle $t a y \rightarrow 0$ as $y \rightarrow a$.

Since angle $x a y \leqq$ angle tax + angle tay, angle $x a y \rightarrow 0$ as $x$ and $y$ both approach $a$. Then Lemma 3 gives the theorem:

10. TheOrem 6. Let $C$ be a simple arc in a convex complete space $Z$ having the four-point property. At a point a let there be a tangent and let $x$ and $y$ be points of $C$ on opposite sides of $a$, with $a x \leqq a y$. Then, as $x$ and $y$ both approach $a,(a x+a y-x y) /(a x) \rightarrow 0$.

Proof. Let the tangent $T$ at $a$ be the union of the unilateral tangents $T^{\prime}$ and $T^{\prime \prime}$ containing points $s$ and $t$, respectively, distinct from $a$. Let $x$ be at the left of $a$ and $T^{\prime}$ be the left-hand tangent. Then angle $x a s \rightarrow 0$ as $x \rightarrow a$ and angle $y a t \rightarrow 0$ as $y \rightarrow a$. By $\$ 5$ the tangent $T$ and the lines determined by $a$ and $x$, and by $a$ and $y$ can be imbedded in $E_{3}$. Consequently angle $x a y \rightarrow \pi$ as $x$ and $y$ both approach $a$. The desired conclusion now follows from $\S 8$.

11. Theorem 7. Let $Z$ be a complete metric space which has the four-point property and is both convex and externally convex. Let $C$ be a simple arc and $a$ be any point not an end-point. Then the conditions of $\$ \$ 9$ and 10 are sufficient for the existence of unilateral and ordinary tangents, respectively.

Proof. Let us take the case of right-hand tangents. Let $x$ and $y$ be any points at the right of $a$ and $C, a x \leqq a y$, and $\theta(x, y)$ be the angle $x a y$. By virtue of the hypothesis, the continuity of the cosine function, and $\S 8$, we have for any positive $\epsilon$ a positive $\delta$ such that

$$
\theta(x, y)<\epsilon / 2 \text { if } a x<\delta \text { and } a y<\delta .
$$

Let $\left\{x_{i}\right\}$ be a sequence of points of $C$ at the right of $a$ and $x_{i} \rightarrow a$ as $i \rightarrow \infty$. For every $i$ greater than some $i^{\prime}, a x_{i}<\delta$. Then (1) becomes 


$$
\theta\left(x_{i}, x_{j}\right)<\epsilon / 2 \text { if } i \geqq i^{\prime} \text { and } j \geqq i^{\prime} \text {. }
$$

Since $Z$ is externally convex there is a point $r_{i}$ on each halfline from $a$ through $x_{i}$ such that $a r_{i}=1$. By (2) and elementary trigonometry the sequence of points $\left\{r_{i}\right\}$ is a Cauchy sequence and converges to a limit $r$ since $Z$ is complete. Let $T$ be the halfline from $a$ through $r$.

Since $r_{i} \rightarrow r$, we have for any positive $\epsilon$ an $i^{\prime \prime}$ for which

$$
\theta\left(r, x_{i}\right)<\epsilon / 2 \text { if } i \geqq i^{\prime \prime} .
$$

Relations (1) and (3) now give $\theta(r, x)<\epsilon$ if $a x<\delta$. Hence $T$ is a right-hand tangent by definition.

Let us now consider the case of the ordinary tangent. In consequence of the first part of the proof we know that, if there is no right-hand tangent, there is a constant $k>0$ and two sequences $\left\{y_{i}\right\}$ and $\left\{y_{i}^{\prime}\right\}$ of points of $C$ at the right of $a$ such that $y_{i} \rightarrow a, y_{i}{ }^{\prime} \rightarrow a$, and

$$
\theta\left(y_{i}, y_{i}^{\prime}\right)>k
$$

From the hypothesis and $\$ 8$ we know that for any positive $\epsilon$, any point $x$ on $C$ at the left of $a$ and sufficiently near to $a$, and $i$ greater than some $i^{\prime}$,

$$
\begin{aligned}
& \pi-\theta\left(x, y_{i}\right)<\epsilon \\
& \pi-\theta\left(x, y_{i}^{\prime}\right)<\epsilon .
\end{aligned}
$$

Taking $\epsilon<k / 4$, we have by (4), (5), and (6)

$$
\theta\left(x, y_{i}\right)+\theta\left(x, y_{i}^{\prime}\right)+\theta\left(y_{i}, y_{i}^{\prime}\right)>2 \pi+k / 2 \text {. }
$$

Since the lines through $a$ and $x, y_{i}, y_{i}{ }^{\prime}$, respectively, can be imbedded in $E_{3}$, relation ( 7$)$ is false.

Thus there is a right-hand and likewise a left-hand tangent. These form a line since $\theta(x, y) \rightarrow \pi$ as $x \rightarrow a$ and $y \rightarrow a$.

The statement and proof of the theorem for the special cases where $a$ is an end-point are obvious.

12. Conclusion. It will be noted that the convergence to zero of $(a x+x y-a y) /(a x)$ when $x$ and $y$ are on the same side of $a$ or of $(a x+a y-x y) /(a x)$ when $x$ and $y$ are on opposite sides of $a$ as $x$ and $y$ approach $a$ on the arc $C$ with $a x \leqq a y$ is an intrinsic 
property of the arc and may be true of a particular simple arc in any metric space. In the first case we may say that $C$ is smooth at the right (left) of $a$, in the second that $C$ is smooth about $a$. The property of smoothness is allied on the one hand to the triangle axiom of metric geometry and on the other to the well known fact from elementary geometry that the distance from a point $x$ on a curve to a tangent at $a$ is an infinitesimal of the second order with respect to $a x$ as $x \rightarrow a$.

For the existence of a tangent line the condition of external convexity or something like it is needed because, as $x \rightarrow a$, the half-line from $a$ through $x$ may shrink to a point, as would be the case if our curve $C$ were a circle and our space $Z$ the union of $C$ and its interior.

In the absence of the four-point property we have the somewhat surprising result that smoothness about a point needs not imply unilateral smoothness. To see this, let us take the following points in the plane: $a=(0,0), b=(-1,0), e=(1,0), f=(1,1)$, $e_{i}=\left(1 / 2^{i}, 0\right), f_{i}=\left(1 / 2^{i}, 1 / 2^{i}\right), i=1,2, \cdots$. Let $Z$ be the union of the segments be, ef, af, $e f_{1},\left\{e_{i} f_{i}\right\}$, and $\left\{e_{i} f_{i+1}\right\}, i=1,2, \cdots$; and let the distance between any two points of $Z$ be the length of the shortest broken line joining them. Then $Z$ is metric, compact, and convex. Now the union of the set $Z-(a f+a e)$ and its limiting points is a simple arc $C$ joining $b$ and $f$. If $x$ and $y$ are points on the opposite sides of $a$, the shortest broken line from $x$ to $y$ passes through $a$; hence $x y=a x+a y$ and so $C$ is smooth about $a$. On the other hand, if $x$ and $y$ are successively the points $\left\{e_{i}\right\}$ and $\left\{f_{i}\right\}$, respectively,

$$
\frac{a x+x y-a y}{a x}=\frac{a e_{i}+e_{i} f_{i}-a f_{i}}{a e_{i}}=\frac{a e_{i}(2-\sqrt{ } 2)}{a e_{i}}=2-\sqrt{ } 2,
$$

and so $C$ is not smooth on the right of $a$.

YALE University 